\title{
A study of different fluid droplets impacting on a liquid film
}

\author{
Huang Qiyu ${ }^{1,2 *}$ and Zhang Holden ${ }^{1}$ \\ ${ }^{1}$ Petroleum Engineering Department, The University of Tulsa, Tulsa, OK 74104, USA \\ ${ }^{2}$ Beijing Key Laboratory of Urban Oil and Gas Distribution Technology, China University of Petroleum, Beijing 102249, \\ China
}

\begin{abstract}
Knowledge of droplet dynamics provides the basis of predicting pressure drops, holdups and corrosion inhibitor distribution in multiphase flow. Droplet size and its distribution also determine the separation efficiency between different phases. Experimental observations were conducted for droplet impingements with different fluids, droplet sizes and velocities, and film thicknesses. The observed transition boundaries were compared with the models developed by different authors. For impingement on a deep pool surface, the Marengo and Tropea correlation for splashing does not agree with the experimental results in this study. The Bai and Gosman critical Weber number for bouncing agrees with the water results but not the oil results. Three new correlations for transition boundaries between bouncing, coalescence, jetting and splashing were proposed and compared with the experimental observations.
\end{abstract}

Key words: Droplet, liquid film, transition boundary, high-speed video

\section{Introduction}

The purpose of this paper is to understand droplet dynamics in multiphase flow. Droplets are formed from films, break from the waves, and are entrained by the gas phase. They also collide with other droplets, break up, coalesce, and impact the films, forming additional droplets or coalescing with the films. Knowledge of droplet dynamics provides the basis of predicting pressure drops, holdups and the distribution of corrosion inhibitor in multiphase flow. Droplet size and its distribution also determine the separation efficiency between different phases. When a droplet impacts on a surface, several kinds of behavior may occur. This depends on the nature of the droplet and the nature of the surface. Bouncing, coalescence, jetting, and splashing have all been reported in the literature. The transitions between these stages have been widely investigated by many researchers. Different correlations for the transitions have been developed. However, there are still many questions unanswered. One is the applicability of these transition models to cover a wide range of operating conditions and fluid properties. The outcome of droplet impact can be significantly different due to different actual conditions. Rein (1993) divided the liquid droplet impact on a liquid film into three categories: bouncing, coalescence and splashing. These three categories are also defined as the three primary transition regimes.

In the bouncing regime, the droplet bounces back during its impact on the surface. It is possible to observe the bouncing of broken droplets whose sizes and velocities

* Corresponding author. email.ppd@cup.edu.cn

Received August 1, 2007 are different from each other. In the coalescence regime, the droplet submerges in the film accompanied with vortex rings. In the splashing regime, a crown is formed at the early stage of splashing. A number of daughter droplets are formed from the tip of the crown. The crown collapses and recoils toward the crown center at the late stage of splashing. This recoiling will eventually form a liquid jet at the center and generate a secondary droplet from the top of the jet. Normally, bouncing and coalescence only occur under fairly low Weber numbers. Rein (1996) further analyzed the possible sub-regimes during the transition from coalescence to splashing using the Weber number as a criterion.

For complicated phenomena, the study of the regime boundaries remains the first priority for a lot of work in the literature. Knowledge of the transition criteria between regimes is critical for the analysis of droplet impact. Several correlations were proposed for prediction of these transitions. Bai and Gosman (1995) collected a number of results from the literature, trying to find a coherent category of the impact phenomena and their thresholds in terms of the dimensionless numbers. For wetted walls with surface temperature lower than boiling temperature, they proposed simple criteria for transitions between bouncing, coalescence and splashing. Cossali et al (1997) investigated the transition from coalescence to splashing by analyzing a large number of pictures. Their correlation was based on the Weber and Ohnesorge numbers, and the liquid film thickness was normalized with the droplet diameter. From detailed experimental studies performed with visualization techniques, Mundo et al (1997; 1998) found a correlation that describes the splashing-deposition (coalescence) limit of an individual droplet impacting on a surface with a defined surface 
roughness height and defined film thickness height. The correlation is a function of Ohnesorge and Reynolds number (or Weber number). Marengo and Tropea (1999) conducted an experimental study and proposed different critical $K$ values for the coalescence-splashing transitions under different surface conditions.

Extensive effort was made to study the liquid droplet impacting on solid or liquid surfaces. Based on these experimental data, several empirical correlations were developed to define/identify the transition regimes. However, each of these correlations was limited to the experimental test matrix on which the correlation was developed. Ideally, a complete set of experimental tests should at least reflect the effects of droplet properties (density, viscosity, and surface tension), droplet size, droplet impact angle and impact velocity, liquid film properties, and liquid film thickness. Several correlations were proposed for predicting these transitions. The commonly used dimensionless parameters to model the regime transition boundaries are the Weber number, Reynolds number, Ohnesorge number, and the relative film thickness.

\section{Experimental}

\subsection{Experimental setup}

Fig. 1 shows the experimental setup. The solid plate was placed inside a transparent tank and submerged under a liquid film. The film thickness was calculated from the liquid volume added into the tank from the liquid level inline with the horizontal plate surface. Liquid droplets with different diameters were produced by using different needles from Gauge 14 to Gauge 33. Correspondingly, the diameter of produced droplets ranged from approximately 4.0 to $1.8 \mathrm{~mm}$. Droplets with a diameter of approximately $1.0 \mathrm{~mm}$ were made by blowing nitrogen gas along the axial direction of a Gauge 33 needle. Needles were connected to a liquid container, and the impact velocity was adjusted by changing the height of the needle above the film surface. The falling droplets and receiving films were the same liquids.

The liquids used in the present study were water and oil of moderate viscosity. Their properties at room temperature are summarized in Table 1.

\subsection{High-speed video system}

A high-speed video system, Photron FASTCAM was used

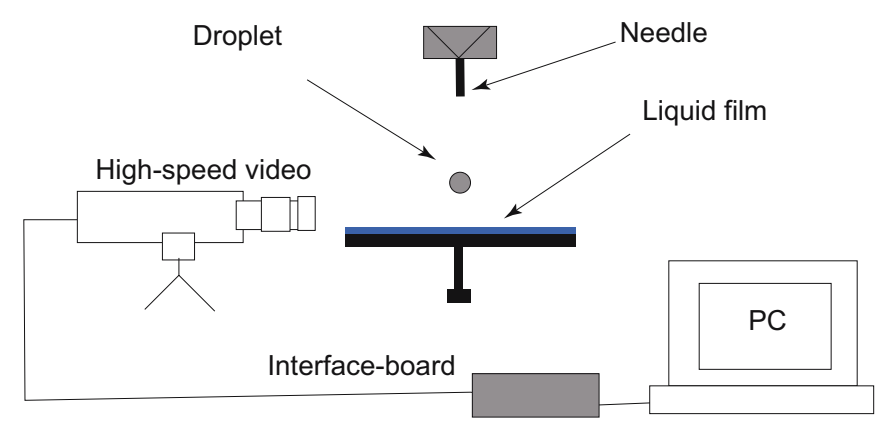

Fig. 1 Experimental setup
Table 1 Properties of liquids used

\begin{tabular}{cccc}
\hline Liquid & $\begin{array}{c}\text { Density } \\
\mathrm{kg} / \mathrm{m}^{3}\end{array}$ & $\begin{array}{c}\text { Viscosity } \\
\mathrm{mPa} \cdot \mathrm{s}\end{array}$ & $\begin{array}{c}\text { Surface tension } \\
\mathrm{N} / \mathrm{m}\end{array}$ \\
\hline Water & 998 & 1.0 & 0.072 \\
Oil & 854 & 22.5 & 0.029 \\
\hline
\end{tabular}

to take high-quality videos and pictures at the moment when a droplet impacted on the film surface. The obtained pictures could be numerically analyzed by a computer program, MATLAB and the size, shape and velocity of the droplets were quantitatively measured. The droplet size was measured by counting the number of pixels occupied by the droplet. The distance corresponding to each pixel was obtained with the recording of the scale at the same place before the droplet impingement test. The droplet impacting velocity was measured with the distance and time corresponding to 6 pixels the droplet traveled before hitting the liquid film. Highspeed video recording is a very good technique for identifying transition boundaries between different kinds of behavior as droplets impinge onto a liquid film.

\section{Results and discussion}

\subsection{Images of different regimes}

Typical images were extracted from the high-speed video records for bouncing, coalescence, jetting and splashing as shown in Figs. 2 through 5, where $\mu$ is the impact velocity of droplet, $D$ is droplet diameter, $\delta$ is the relative film thickness, $\delta=\frac{h}{D}$.

\subsection{Impingement on deep pool surface}

Splashing, jetting, coalescence and bouncing were observed when a droplet impacted vertically on the surface of a deep pool of water or oil. Based on Marengo and Tropea's (1999) classification, the liquid thickness of 10 and $140 \mathrm{~mm}$ used in this study could be categorized as a deep pool. Using logarithmic coordinates, Fig. 6 shows the experimental observations of the above reacting phenomena and the comparisons with the available transition criteria found in the literature. Marengo and Tropea's correlation (1999) can be written as follows:

$$
W e \cdot O h^{-0.4}=8000
$$

where $W e$ is the Weber number; $O h$ is the Ohnesorge number.

For the transition between coalescence and splashing, Eq. (1) does not agree well with experimental results. The following correlation for the transition between coalescence and splashing gives a better fitting with the experimental observations:

$$
W e^{0.375} \cdot R e^{0.25}=70
$$

where $R e$ is the Reynolds number.

The Hsiao et al model for jetting (1988) is also plotted in Fig. 6. This model agrees with experimental observations 


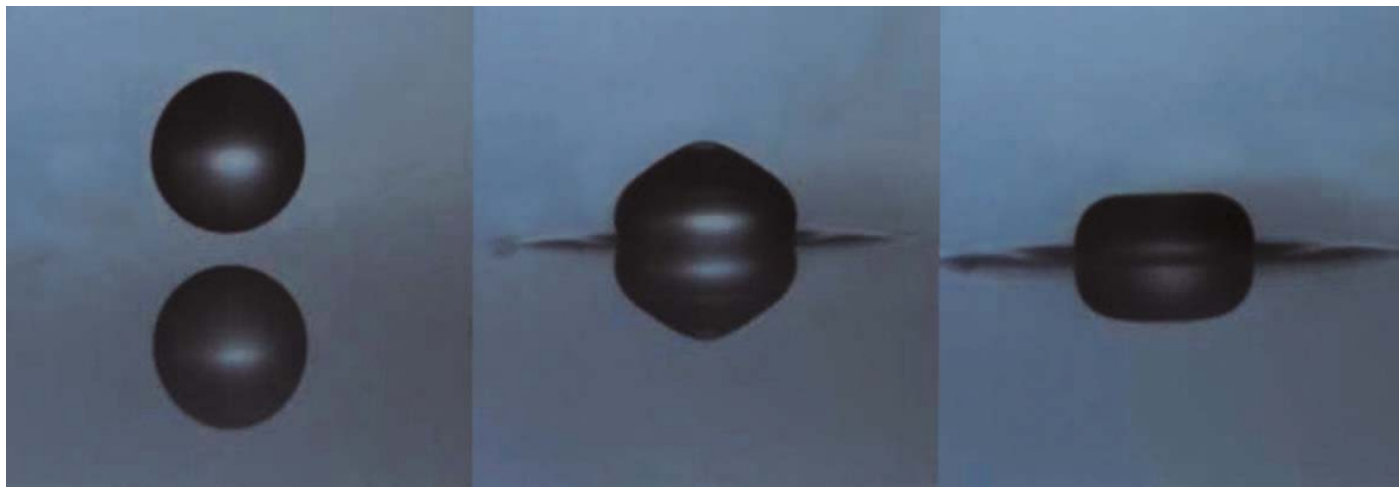

(a)

(b)

(c)

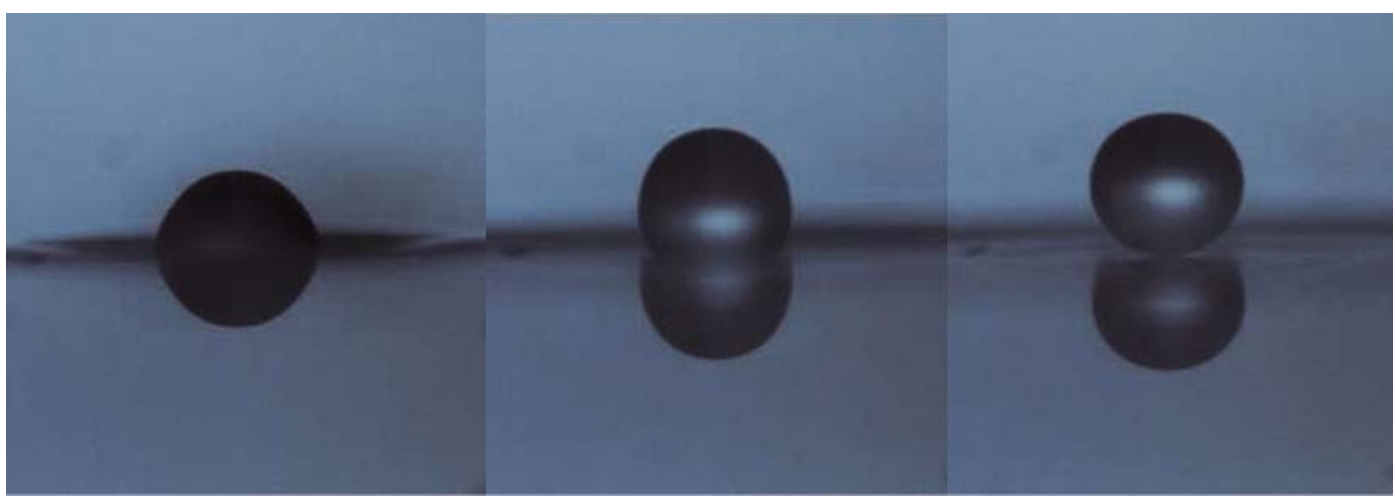

(d)

(e)

(f)

Fig. 2 Bouncing (water, $D=2 \mathrm{~mm}, u=26.4 \mathrm{~cm} / \mathrm{s}, \delta=70$ )

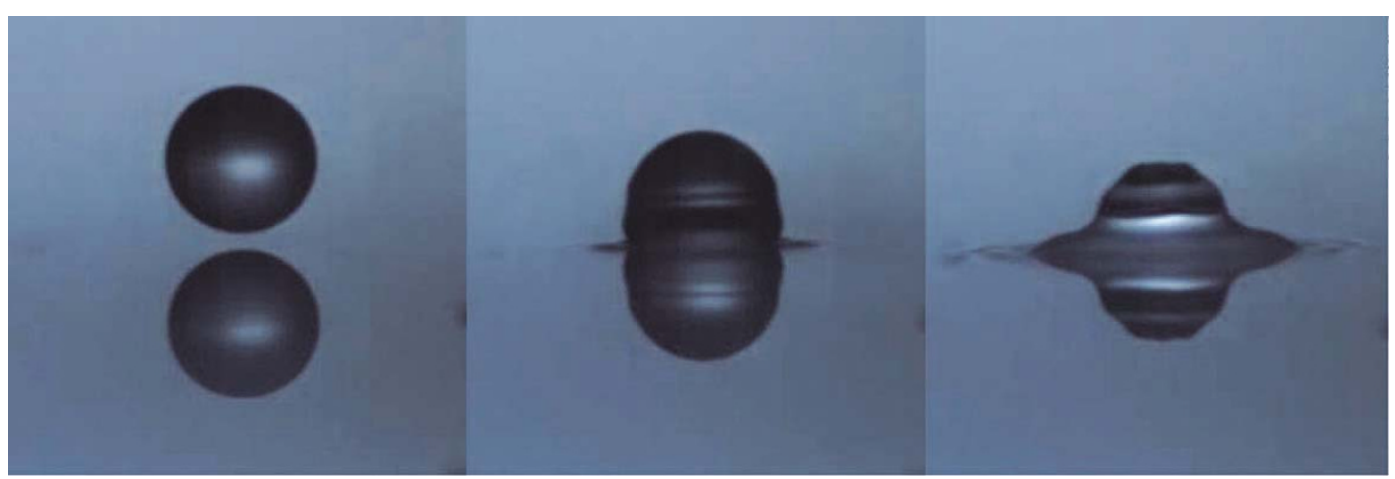

(a)

(b)

(c)

Fig. 3 Coalescence (water, $D=2 \mathrm{~mm}, u=34.1 \mathrm{~cm} / \mathrm{s}, \delta=70$ )
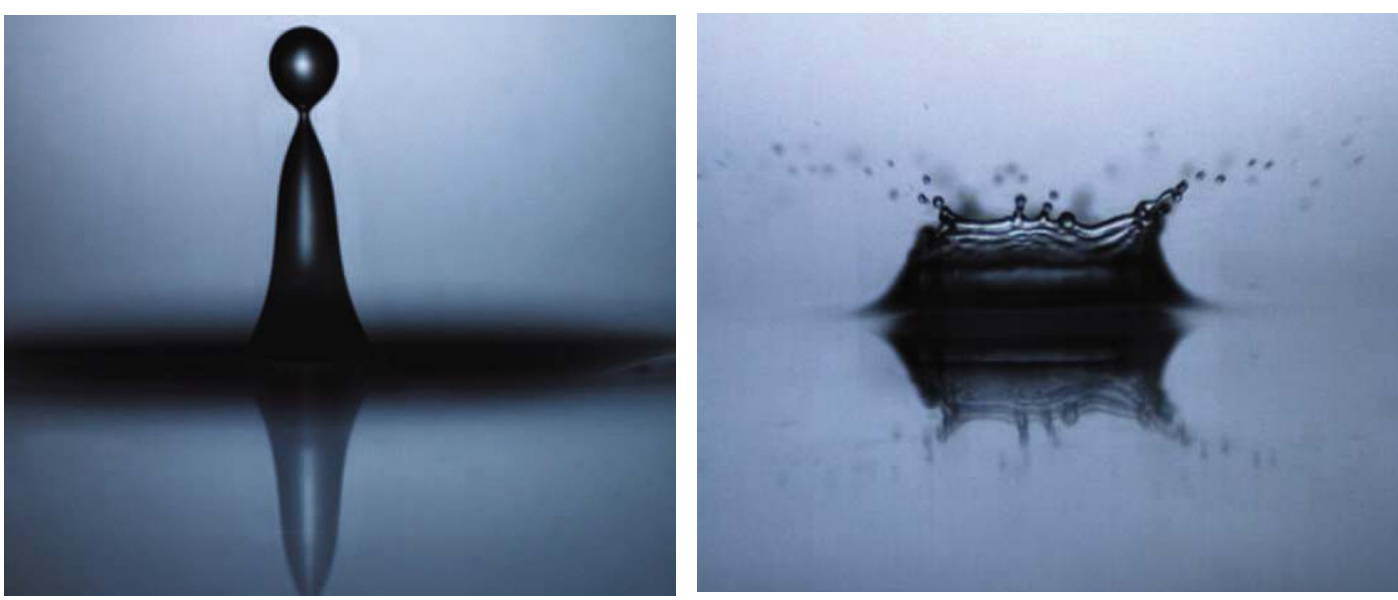

Fig. 4 Jetting (water, $D=2 \mathrm{~mm}, u=290.2 \mathrm{~cm} / \mathrm{s}, \delta=70$ ) Fig. 5 Splashing (water, $D=2 \mathrm{~mm}, u=338.0 \mathrm{~cm} / \mathrm{s}, \delta=70$ ) 


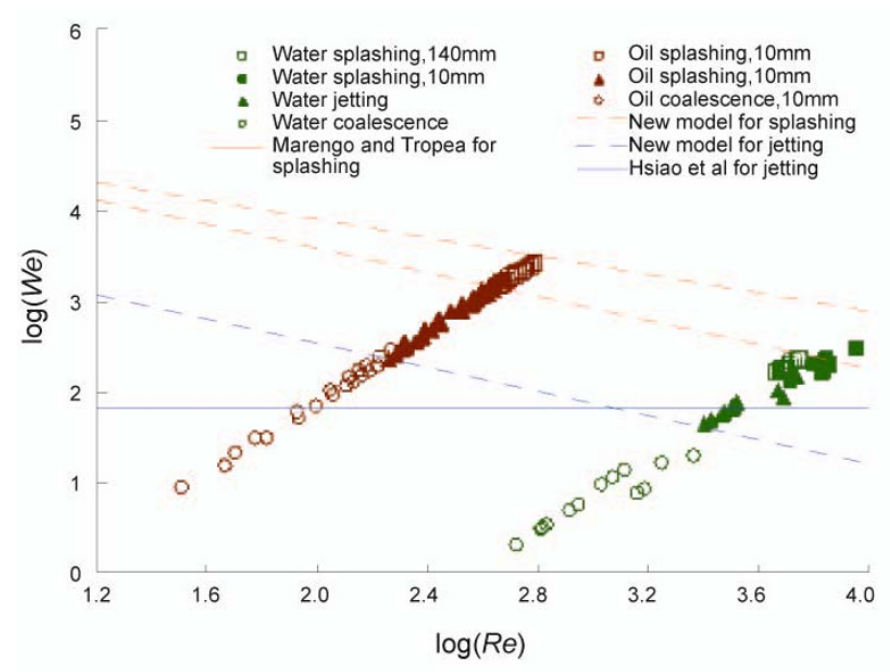

Fig. 6 Splashing and jetting on liquid pool surface

when water is used as the fluid. Experimental results showed that the coalescence-jetting transition also depended on the viscosity of the fluid, which was not included in the Weber number. A new correlation was proposed for this transition boundary and also plotted in Fig. 6:

$$
W e^{0.375} \cdot R e^{0.25}=28
$$

Fig. 7 shows observations of the transition between bouncing and coalescence of a droplet impacting on a liquid pool surface. Bai and Gosman's (1995) criterion for bouncing is also plotted in Fig. 7, which corresponds to a constant Weber number of 5. However, the observations also indicated a dependence on Reynolds number. The following correlation can be used as a rough guideline to predict the bouncingcoalescence transition:

$$
W e^{0.375} \cdot R e^{0.25}=13
$$

As seen in Fig. 7, the transition boundary between bouncing and coalescence is not identified as clearly as for jetting and splashing. This may be due to the deformation of droplets. The needle must be kept very close to the film surface to ensure a very low droplet velocity around the

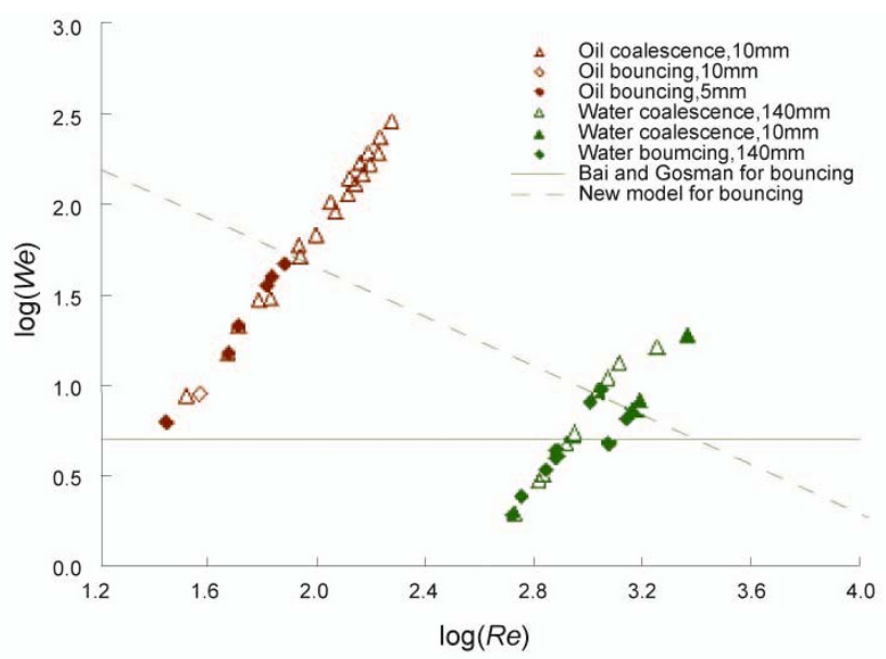

Fig. 7 Bouncing on liquid pool surface transition. When the droplet was released from the needle tip, its shape began to oscillate. The droplet could not resume the spherical shape when it hit the film surface because of the short distance. In this study, the low Weber number and Reynolds number could not be obtained by reducing the droplet diameter to less than $1 \mathrm{~mm}$. Smaller droplets could not be produced even if a very thin needle was used due to the attachment of the droplet around the needle tip.

\subsection{Impingement on film surface}

The transition between coalescence and splashing was observed for the impingement of droplets on a water or oil film. Film thicknesses of 1 and $3 \mathrm{~mm}$ were used. The experimental results were compared with predicted results from Cossali et al model (1997) in Fig. 8. The model agreed with experimental results only for the $1 \mathrm{~mm}$ oil film. Significant differences existed between the model predicted and the experimentally observed transition boundaries for the $3 \mathrm{~mm}$ oil film and the water films.

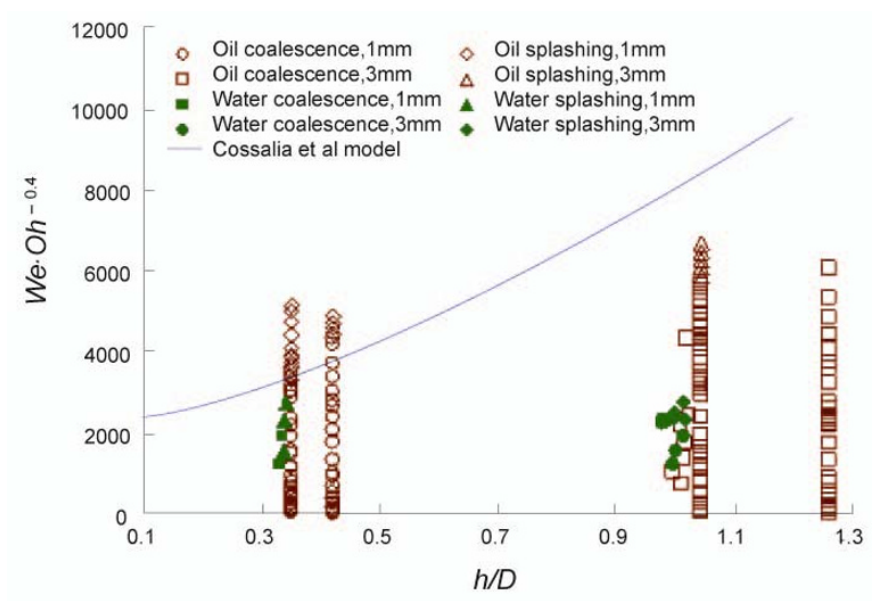

Fig. 8 Coalescence-splashing transition on film compared with Cossalia et al model (1997)

Marengo and Tropea (1999) employed two different correlations to predict the transitions on liquid films of water and fluid with viscosity higher than $50 \mathrm{mPa} \cdot \mathrm{s}$ :

For water

$$
W e \cdot O h^{-0.4}=2074+870 \delta^{0.23}
$$

For $\mu>50 \mathrm{mPa} \cdot \mathrm{s}$ and $O h>0.01$

$$
W e \cdot O h^{-0.4}=2164+7560 \delta^{1.78}
$$

These two correlations were compared with experimental observations in Fig. 9 for oil droplet impingement on liquid film. The observed coalescence-splashing transition boundary located between the predictions of these two correlations showing an agreement considering the oil viscosity of this study $(22.5 \mathrm{mPa} \cdot \mathrm{s})$. Fig. 10 shows that the observed transition boundary is slightly lower than the model prediction for water droplet impingement.

Based on the observations of the droplet impingements on thin films of water and oil, the following correlation was proposed to predict the coalescence-splashing transition:

$$
(W e \cdot R e)^{0.25}=25+78^{1.44}
$$


The predictions using this correlation were compared with experimental results in Fig. 11. Good agreements were observed for impingements on both oil and water films.

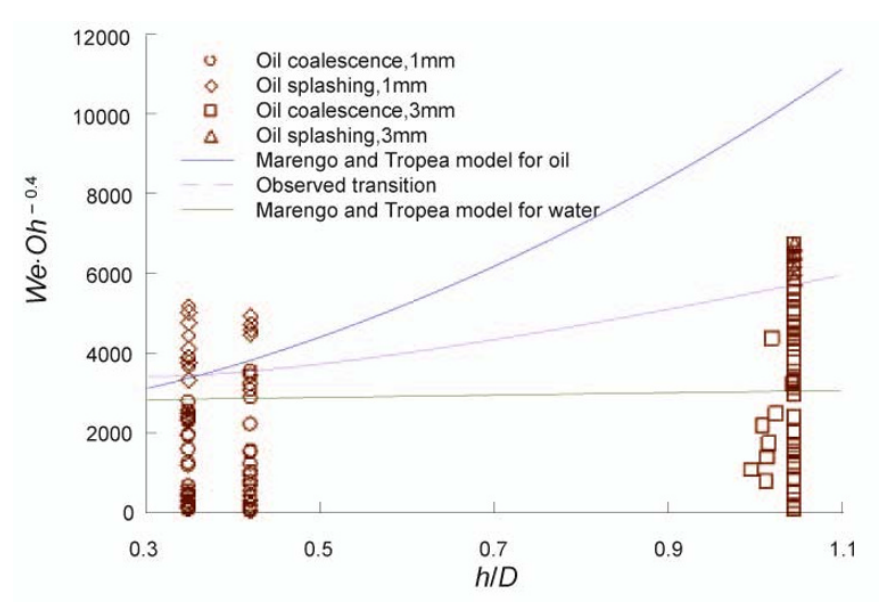

Fig. 9 Coalescence-splashing transition on oil film (Compared with Marengo and Tropea (1999))

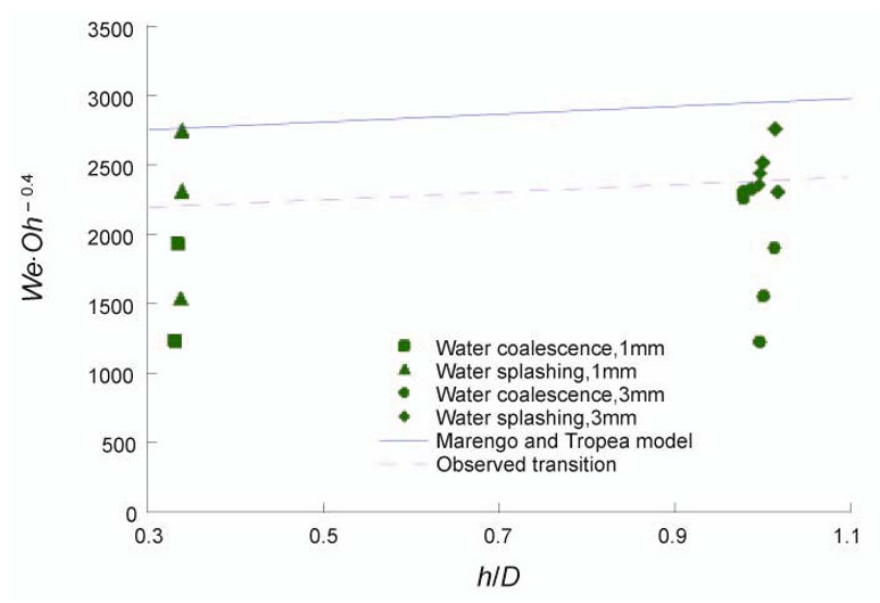

Fig. 10 Coalescence-splashing transition on water film (Compared with Marengo and Tropea (1999) )

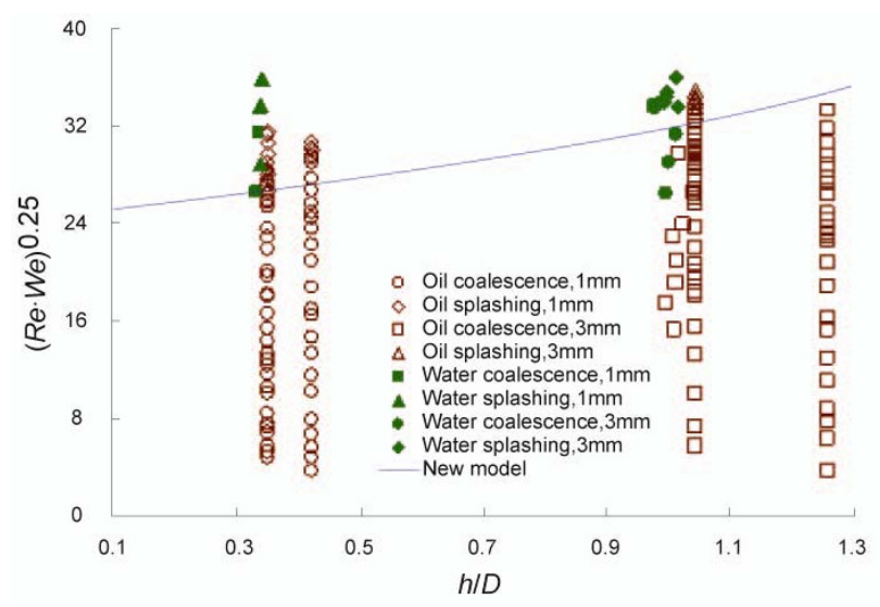

Fig. 11 Coalescence-splashing transition on film (Compared with new model)
Jetting is restricted by the liquid film thickness. With a $1 \mathrm{~mm}$ liquid film, the collapse into the center after the impingement can not form a thin liquid jet, which then produces secondary droplets. Instead, only a liquid bulge is formed before dissipating into small amplitude waves propagating outward.

\section{Conclusions}

1) Experimental observations have been conducted for droplet impingements with different fluids, droplet sizes and velocities, and film thicknesses. High-speed video recording is shown to be an effective technique for identifying the transition boundaries between bouncing, coalescence, jetting and splashing.

2) The observed transition boundaries were compared with the models developed by different authors. For impingement on deep pool surface, the Marengo and Tropea correlation for splashing did not agree with the experimental results in this study. The Hsiao et al critical Weber number for jetting and the Bai and Gosman critical Weber number for bouncing agreed with the water results but not the oil results. Three new correlations for transition boundaries between bouncing, coalescence, jetting and splashing were proposed and compared with the experimental observations.

3) For the impingement on a liquid film surface, the Cossali et al model and the Marengo and Tropea model for the splashing-coalescence transition compared well with the thin oil film $(1 \mathrm{~mm})$ data, but the comparisons with water film data were not good. A new correlation was also proposed for liquid film surface impingement.

\section{References}

Bai C and Gosman A D. Development of methodology for spray impingement simulation. Society of Automotive Engineers. 1995. 104(33): 550-568

Cossali G E, Brunello G, Coghe A, et al. Impact of a single drop on a liquid film: Experimental analysis and comparison with empirical models. Italian Congress of Thermofluid Dynamics UIT, Ferrara, 30 June-2 July, 1999

Cossali G E, Coghe A and Marengo M. The impact of a single drop on a wetted solid surface. Experiments in Fluids. 1997. 22: 463-472

Hsiao M, Lichter S and Quintero L G. The critical Weber number for vortex and jet formation for drops impinging on a liquid pool. Physics of Fluids. 1988. 31(12): 3560-3562

Marengo M and Tropea C. Aufprall von Tropfen auf Flüssigkeitsfilme. $\operatorname{Tr}$ 194/10 1, 2, Deutsche Forschungsgemeinschaft. 1999.

Mundo C, Sommerfeld M and Tropea C. On the modeling of liquid sprays impinging on surfaces. Atomization and Sprays. 1998. 8(6): 625-652

Mundo C, Tropea C and Sommerfeld M. Numerical and experimental investigation of spray characteristics in the vicinity of a rigid wall. Experimental Thermal and Fluid Science. 1997. 15(3): 228-237

Rein M. Phenomena of liquid drop impact on solid and liquid surfaces. Fluid Dynamics Research. 1993. 12: 61-93

Rein M. The transitional regime between coalescing and splashing drops. Journal of Fluid Mechanics. 1996. 306: 145-165

(Edited by Sun Yanhua) 